\title{
Low-energy fusion dynamics of weakly bound nuclei: A time dependent perspective
}

\author{
A. Diaz-Torres and M. Boselli
}

ECT*, Strada delle Tabarelle 286, 38123 Villazzano, Trento, Italy

\begin{abstract}
Recent dynamical fusion models for weakly bound nuclei at low incident energies, based on a time-dependent perspective, are briefly presented. The main features of both the PLATYPUS model and a new quantum approach are highlighted. In contrast to existing timedependent quantum models, the present quantum approach separates the complete and incomplete fusion from the total fusion. Calculations performed within a toy model for ${ }^{6} \mathrm{Li}+{ }^{209} \mathrm{Bi}$ at near-barrier energies show that converged excitation functions for total, complete and incomplete fusion can be determined with the time-dependent wavepacket dynamics.
\end{abstract}

\section{Introduction}

Nuclear reactions constitute the engine of stars and control nucleosynthesis. They are the key tool for extracting the properties of exotic nuclei at rare-isotope beam facilities. The physics of low-energy nuclear reactions is exciting because it integrates many-body nuclear structure, reaction dynamics and mechanisms as well as quantum mechanics.

The present contribution addresses the fusion dynamics of weakly bound nuclei from a time-dependent perspective which is interesting, as it provides an intuitive picture of the reaction dynamics and how reaction observables are set during a nuclear collision. The question is how two complex nuclei 


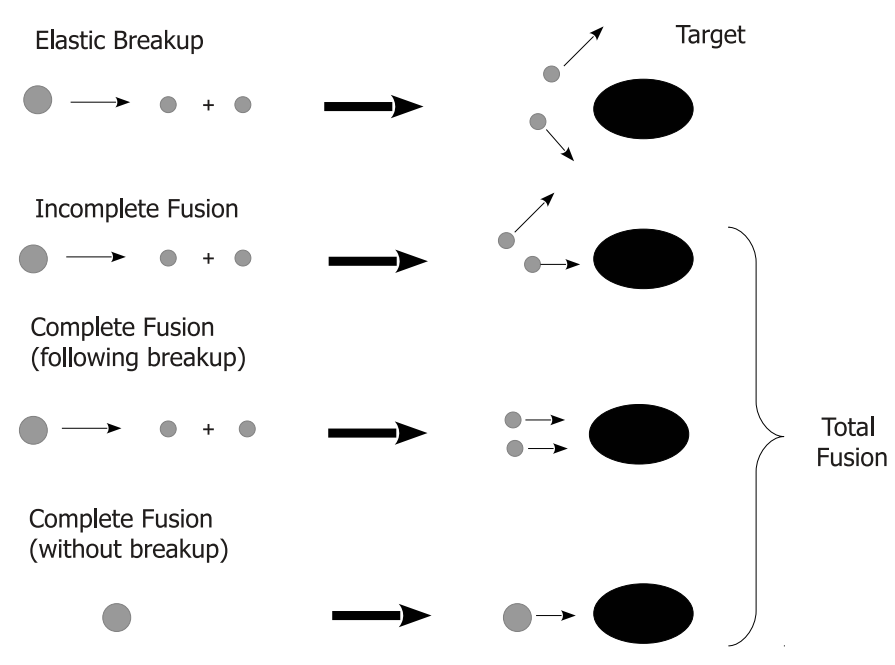

Figure 1: Some reaction processes of a weakly-bound two-body projectile.

collide and the reaction observables emerge when the nuclei pass each other slowly. There is spectacular experimental evidence that nuclear structure and reaction dynamics are strongly coupled, the coupling determining the reaction outcomes [1].

Most of the low-lying states of weakly-bound nuclei have a few-body cluster structure. A few states are bound and most of the spectrum lies in the continuum of scattering states. The strong coupling to that continuum is essential for understanding the reaction dynamics. These fragile structures dissociate when they collide with stable targets, as illustrated in Fig. 1. Many reaction paths emerge, such as elastic breakup and various fusion processes. In the incomplete fusion (ICF) only a part of the projectile is captured by the target, while in the complete fusion $(\mathrm{CF})$ all the projectile fragments are captured. The sum of ICF and CF defines the total fusion (TF). It is a challenge to describe all these reaction processes in a unified picture as well as to measure all the cross sections unambiguously. To separate the fusion processes is a great problem as well. Different approaches have been used to investigate these issues [2]. A classical and a quantum mechanical model, which are based on a time-dependent perspective, are discussed below. 


\section{Selected dynamical fusion models}

\section{$2.1 \quad$ The PLATYPUS model}

The PLATYPUS model uses two notions: classical trajectory and MonteCarlo sampling of probability densities. This approach has been developed in close collaboration with experimenters $[3,4]$. The model is implemented in the PLATYPUS code [5].

For a given impact parameter and bombarding energy, a weakly bound two-body projectile describes an orbit on which the projectile can be dissociated by the projectile-target interaction. A crucial input of this model is an experimentally determined breakup function, constructed as follows. For instance, if a ${ }^{9} \mathrm{Be}(=2 \alpha+\mathrm{n})$ dissociates on a stable target, the alpha particles can be detected in coincidence for a given scattering angle of ${ }^{9} \mathrm{Be}$. The breakup cross section is divided by the Rutherford scattering cross section, defining a breakup probability as a function of the distance of closest approach $R_{\min }$. This probability is interpreted as a total breakup probability, which results from the integration of a local probability density over the projectile-target distances [4]. The observed breakup function of weaklybound stable nuclei $\left({ }^{9} \mathrm{Be},{ }^{6,7} \mathrm{Li}\right)$ appears to be a linear function of $R_{\min }$ in logarithmic scale $[6,7]$, whose slope is the same as that of the local probability density. Other probability densities are employed in the PLATYPUS model, which can be found in Refs. [3,4]. The final destination of the projectile fragments (captured or non-captured by the target) is determined solving classical equations of motion. Based on those trajectories, probabilities and (integrated and differential) cross sections are constructed. The PLATYPUS model connects the sub-Coulomb breakup process to the above-barrier $\mathrm{CF}$ and ICF processes $[3,4]$.

Figure 2 shows the above-barrier $\mathrm{CF}$ excitation function for ${ }^{7} \mathrm{Li}+{ }^{198} \mathrm{Pt}$ (solid curve). It is observed that the sequential fusion component (dashed curve) becomes important as energy increases. This CF component (CF following breakup in Fig. 1) is usually neglected in quantum mechanical models, but should be included for describing the CF precisely. For this system, the ICF has also been studied with the PLATYPUS model, as reported in Refs. $[8,9]$. PLATYPUS provides useful information (angular momentum and excitation energy distributions) on the primary ICF products along with differential cross sections of the surviving breakup fragment [9]. The interaction between the fusing fragment and the survival fragment is very important, as this interaction affects both the angular momentum and kinetic energy brought by the captured fragment into the target. These 


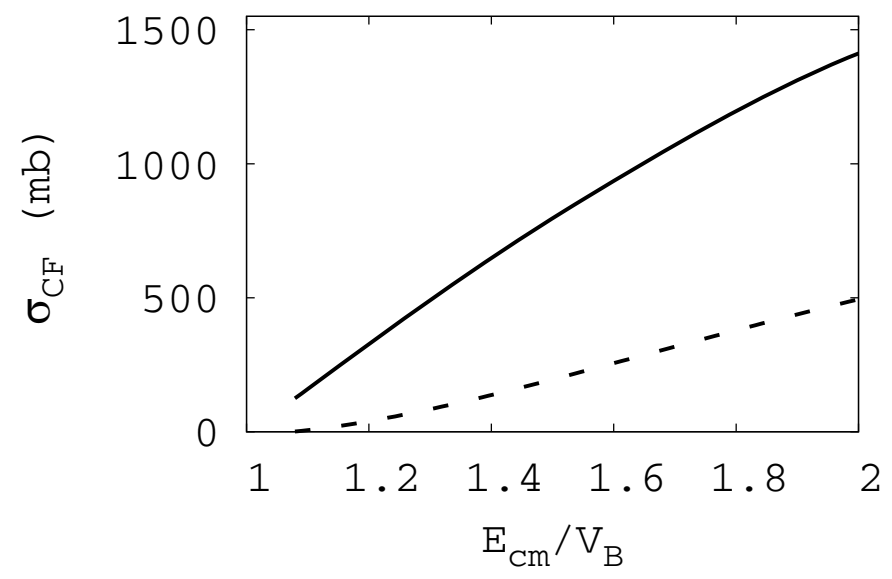

Figure 2: The above-barrier CF excitation function (solid curve) for ${ }^{7} \mathrm{Li}+{ }^{198} \mathrm{Pt}$ from the PLATYPUS model. The sequential fusion component (dashed curve) becomes important as energy increases.

observables are useful for planning and interpreting particle-gamma coincidence measurements [8]. In Ref. [8], the spin and excitation energy distributions from PLATYPUS are used in combination with the evaporation code PACE for calculating the production of specific evaporation residues. Some evaporation residue cross sections can be explained by the breakup-fusion mechanism implemented in the PLATYPUS model.

Dedicated sub-Coulomb breakup measurements, involving the ${ }^{6,7} \mathrm{Li}$ and ${ }^{9}$ Be projectiles, have revealed that the projectile fragmentation is triggered by transfer dominantly $[7,10]$. This process enriches the fusion scenario of weakly bound nuclei, motivating the development of more comprehensive fusion models. As an example, Fig. 3 presents a preliminary PLATYPUS calculation of ICF for ${ }^{7} \mathrm{Li}+{ }^{209} \mathrm{Bi}$ at $E_{c m} / V_{B}=1.2$. It shows the contribution of the observed fragmentation processes of ${ }^{7} \mathrm{Li}$ to the total ICF cross section. The contribution of the ${ }^{8} \mathrm{Be}$ mediated breakup yield on ICF is much larger than the contribution of the direct ${ }^{7} \mathrm{Li}$ breakup into alpha and triton. Some projectile-like nuclei $\left({ }^{5} \mathrm{Li},{ }^{8} \mathrm{Be}\right)$ are unbound in their ground states, so these resonant states may change the breakup location of these nuclei with respect to the target position. Only prompt breakup near the target may affect the $\mathrm{CF}$ and ICF cross sections. The understanding of the role of the breakup-triggered-by-transfer process in fusion seems to be important for 


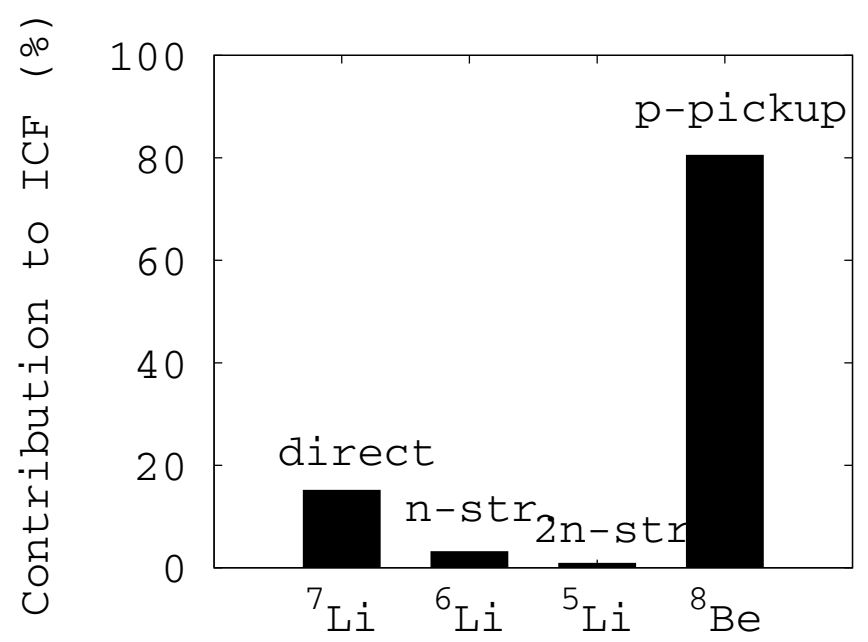

Figure 3: The contribution of various ${ }^{7} \mathrm{Li}$ fragmentation processes to the ICF for ${ }^{7} \mathrm{Li}+{ }^{209} \mathrm{Bi}$ at $E_{c m} / V_{B}=1.2$, calculated with the PLATYPUS model.

making further progress in this field.

\subsection{Time-dependent wave-packet dynamics}

A quantum dynamical approach is being developed [9] using the timedependent wave-packet (TDWP) method. This model can deal with quantum tunneling, making it possible to investigate the interplay of breakup and fusion at sub-Coulomb energies. This perspective has been already employed in the past, see e.g. Ref. [11]. The method is based on three key steps [12]:

(i) Preparation of the initial three-body wave function, $\Psi(t=0)$,

(ii) Time propagation, $\Psi(t)=\exp (-i \hat{\mathcal{H}} t / \hbar) \Psi(0)$, where $\hat{\mathcal{H}}$ is the total three-body Hamiltonian,

(iii) Analysis of the long-time propagated wave function, $\Psi\left(t_{f}\right)$, to calculate energy-resolved cross sections.

\subsubsection{A one-dimensional toy model with two degrees of freedom}

As a starting point, we have studied the ${ }^{6} \mathrm{Li}+{ }^{209} \mathrm{Bi}$ fusion within a onedimensional model with two degrees of freedom, where the ${ }^{209} \mathrm{Bi}$ target and 
the ${ }^{6} \mathrm{Li}$ fragments $\left({ }^{4} \mathrm{He}\right.$ and $\left.{ }^{2} \mathrm{H}\right)$ are always on a line [9]. $X_{C M}$ denotes the distance between the target and the center of mass of the projectile, whereas $\xi$ gives the distance between the projectile fragments. $M$ is the mass of the target nucleus, while $m_{1}$ and $m_{2}$ are the masses of the projectile fragments. The Hamiltonian of the system in terms of these coordinates reads:

$$
\hat{\mathcal{H}}=\frac{\hat{P}_{X_{C M}}^{2}}{2 \mu_{T P}}+\frac{\hat{p}_{\xi}^{2}}{2 \mu_{12}}+U_{12}(\xi)+V_{T 1}\left(X_{C M}-\alpha \xi\right)+V_{T 2}\left(X_{C M}+\beta \xi\right)
$$

where $\mu_{T P}=M\left(m_{1}+m_{2}\right) /\left(M+m_{1}+m_{2}\right), \mu_{12}=m_{1} m_{2} /\left(m_{1}+m_{2}\right)$, $\alpha=m_{2} /\left(m_{1}+m_{2}\right), \beta=m_{1} /\left(m_{1}+m_{2}\right)$. $U_{12}$ represents the interaction between the fragments. $V_{T 1}$ and $V_{T 2}$ describe the interaction between target and the two projectile fragments, respectively, which depend on the relative distances $x_{1}=X_{C M}-\alpha \xi$ and $x_{2}=X_{C M}+\beta \xi$. In the calculations below, Woods-Saxon nuclear potentials are used for the binary systems, while for their Coulomb interaction the potential of a uniformly charged sphere is employed.

To describe fusion of the projectile fragments with the target, the Hamiltonian (1) is augmented with two strong imaginary potentials, $i W_{T 1}\left(x_{1}\right)$ and $i W_{T 2}\left(x_{2}\right)$, which operate in the interior of the individual Coulomb barriers between the target and the projectile fragments. This is usually employed in the coupled-channels model of fusion [1]. The imaginary potentials have a Woods-Saxon shape, centered at the minimum of the individual potential pockets.

Initial wave function. Since at the initial time the projectile is considered to be far apart from the target, $V_{T 1}$ and $V_{T 2}$ in Eq. (1) can be neglected and the Hamiltonian becomes separable. Consequently, the initial wave function can be factorized as

$$
\Psi\left(\xi, X_{C M}, t=0\right)=\Phi_{0}\left(X_{C M}\right) \chi_{0}(\xi),
$$

where $\chi_{0}$ describes the ground state of the projectile, while $\Phi_{0}\left(X_{C M}\right)$ is chosen to be a Gaussian wave-packet centered in $X_{0}$, with spatial width $\sigma$ and average energy $E_{0}$. The ${ }^{6} \mathrm{Li}\left(={ }^{4} \mathrm{He}+{ }^{2} \mathrm{H}\right)$ wave function is calculated using a nuclear Woods-Saxon potential between the alpha particle and the deuteron, which provides a $1 s$-state with a separation energy of $-1.47 \mathrm{MeV}$. Please note that the $X_{C M}$ and $\xi$ coordinates are strongly coupled when the ${ }^{6} \mathrm{Li}$ and ${ }^{209} \mathrm{Bi}$ nuclei come together, so a product state (2) is only justified asymptotically.

Time propagation. The time evolution operator is represented as a convergent series of modified Chebyshev polynomials $[12,13]$. In the absence of 
the imaginary potentials, the norm of the wave function is preserved with an accuracy of $\sim 10^{-14}$.

Analysis. A limitation of most fusion models of weakly bound nuclei is the lack of an unambiguous calculation of $\sigma_{C F}$ and $\sigma_{I C F}$ [9]. We think that a key idea to overcome this problem is to examine the location of each fragment with respect to the position of the individual Coulomb barriers, irrespective of the internal excitation of the ${ }^{6} \mathrm{Li}$ projectile: if a fragment is located inside the radius of its Coulomb barrier it is considered captured by the target. Otherwise, it is not captured. We identify a CF event when both fragments are located inside their individual barrier radii, while an ICF event occurs when just one of the fragment is inside its barrier radius. This idea is realized by means of position projection operators, as explained in detail in Ref. [9].

The energy-resolved fusion cross section can be expressed in terms of the energy-resolved transmission coefficient through the Coulomb barrier:

$$
\sigma(E)=\frac{\pi \hbar^{2}}{2 \mu E} \mathcal{T}(E)
$$

where each fusion process (TF, ICF and $\mathrm{CF}$ ) has its own transmission coefficient $\mathcal{T}(E)$.

The energy-resolved transmission coefficients can be determined using the window operator method [14]. In the following discussion, $\Psi$ refers to a wave function which has any asymptotic contribution at large $X_{C M}$, such as the initial and final total wave functions as well as the parts of the total wave function describing the ICF and the scattering process [9]. The main idea is, for instance, to calculate the energy spectrum, $\mathcal{P}\left(E_{k}\right)$, of the initial and final wave functions. $E_{k}$ is the centroid of a total energy bin of width $2 \epsilon$. A vector of reflection coefficients, $\mathcal{R}\left(E_{k}\right)$, is determined by the ratio [11,12]:

$$
\mathcal{R}\left(E_{k}\right)=\frac{\mathcal{P}^{\text {final }}\left(E_{k}\right)}{\mathcal{P}^{\text {initial }}\left(E_{k}\right)} .
$$

The transmission coefficients are:

$$
\mathcal{T}\left(E_{k}\right)=1-\mathcal{R}\left(E_{k}\right) .
$$

The energy spectrum $\mathcal{P}\left(E_{k}\right)=\langle\Psi|\hat{\Delta}| \Psi\rangle$, where $\hat{\Delta}$ is the window operator [14]:

$$
\hat{\Delta}\left(E_{k}, n, \epsilon\right) \equiv \frac{\epsilon^{2^{n}}}{\left(\hat{\mathcal{H}}_{\text {asym }}-E_{k}\right)^{2^{n}}+\epsilon^{2^{n}}},
$$




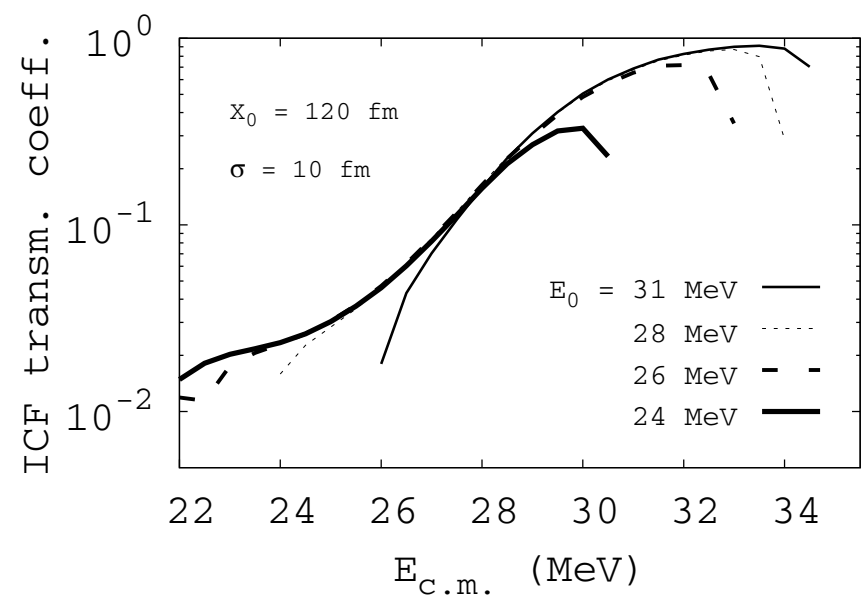

Figure 4: Energy-resolved ICF excitation function from the TDWP method. A converged curve can be constructed.

$\hat{\mathcal{H}}_{\text {asym }}$ is the asymptotic part of the system Hamiltonian $\hat{\mathcal{H}}$, and $n$ determines the shape of the window function. As $n$ is increased, this shape rapidly becomes rectangular with very little overlap between adjacent energy bins [14], the bin width remaining constant at $2 \epsilon$. The spectrum is constructed for a set of $E_{k}$ where $E_{k+1}=E_{k}+2 \epsilon$. Thus, scattering information over a range of incident energies can be extracted from a time-dependent wave function that has been calculated on a grid. In this work, $n=2$ and $\epsilon=0.25$ $\mathrm{MeV}$. Solving two successive linear equations for the vector $|\chi\rangle$ :

$$
\left(\hat{\mathcal{H}}_{\text {asym }}-E_{k}+\sqrt{i} \epsilon\right)\left(\hat{\mathcal{H}}_{\text {asym }}-E_{k}-\sqrt{i} \epsilon\right)|\chi\rangle=|\Psi\rangle,
$$

yields $\mathcal{P}\left(E_{k}\right)=\epsilon^{4}\langle\chi \mid \chi\rangle$.

Calculations of energy-resolved transmission coefficients (fusion probabilities) have been performed using a Fourier grid in the $\left(\xi, X_{C M}\right)$ coordinates [12]: $\xi=0-30 \mathrm{fm}$ and $X_{C M}=0-200 \mathrm{fm}$ with 128 and 256 evenly spaced points, respectively.

As an example, Fig. 4 shows the energy-resolved ICF probability and its dependence on the average energy $E_{0}$ of the initial wave-packet. We have checked [15] the convergence of the calculations regarding the $X_{0}$ and $\sigma$ parameters of the initial wave packet for a broad range of values around those presented in Fig. 4. A few wave-packet propagations allows one to calculate the converged ICF excitation function in a broad range of incident 
energies. In contrast to the usual CDCC fusion calculations [17-19], this ICF excitation function includes ICF from the projectile bound state(s) [20].

\section{Summary}

The time-dependent perspective is useful for understanding and quantifying low-energy reaction dynamics of exotic nuclei. It finds realization in the classical and quantum mechanical realms. PLATYPUS is a powerful tool for planning and interpreting (fusion and breakup) measurements, which allow its fine tuning. A quantum approach to low-energy reaction dynamics of weakly-bound few-body nuclei, based on the time-dependent wave-packet method, is being developed. It incorporates novel features into existing models based on this perspective, such as the separation of the complete and incomplete fusion from the total fusion. This type of approach is powerful because (i) it provides an intuitive description of the reaction dynamics, and (ii) all the continuum couplings are included. We have implemented this approach in a one-dimensional reaction model with two degrees of freedom. A method for an unambiguous calculation of complete and incomplete fusion cross sections is used, which includes incomplete fusion from bound states as well as complete fusion from unbound states of the weakly bound projectile. Model calculations show that converged reliable fusion excitation functions can be obtained with the time-dependent wave-packet method. This approach is being developed further, using a three-dimensional model. Additional cross sections, such as elastic, breakup and transfer cross sections, can also be calculated within this approach. Understanding the breakup mechanisms and their impact on unambiguously separated complete and incomplete fusion processes could make for further progress in the field. The video of this presentation can be seen in Ref. [16].

\section{References}

[1] Back B.B., Esbensen H., Jiang C.L. and Rehm K.E., Rev. Mod. Phys., 86 (2014) 317.

[2] Canto L.F., Gomes P.R.S., Donangelo R., Lubian J. and Hussein M.S., Phys. Rep., (2015) in press.

[3] Diaz-Torres A., Hinde D.J., Tostevin J.A., Dasgupta M. and Gasques L.R., Phys. Rev. Lett., 98 (2007) 152701. 
[4] Diaz-Torres A., J. Phys. G, 37 (2010) 075109.

[5] Diaz-Torres A., Comp. Phys. Comm., 182 (2011) 1100.

[6] Ramin R. et al., Phys. Rev. C, 81 (2010) 024601.

[7] Luong D.H. et al., Phys. Rev. C, 88 (2013) 034609.

[8] Shrivastava A. et al., Phys. Lett. B, 718 (2013) 931.

[9] Boselli M. and Diaz-Torres A., J. Phys. G, 41 (2014) 094001.

[10] Luong D.H. et al., Phys. Lett. B, 695 (2011) 105.

[11] Yabana K., Prog. Theor. Phys., 97 (1997) 437.

[12] Tannor D.J., in Introduction to Quantum Mechanics: A TimeDependent Perspective (University Science Books, Saulito, California) 2007, pp. 273-331.

[13] Mandelshtam V.A. and Taylor H.S, J. Chem. Phys., 103 (1995) 2903.

[14] Schafer K.J. and Kulander K.C., Phys. Rev. A, 42 (1990) 5794.

[15] Boselli M. and Diaz-Torres A., in preparation.

[16] https://www.youtube.com/watch?v=gLe-RxC8hZU.

[17] Hagino K., Vitturi A., Dasso C.H. and Lenzi S.M., Phys. Rev. C, 61 (2000) 037602.

[18] Diaz-Torres A. and Thompson I.J., Phys. Rev. C, 65 (2002) 024606.

[19] Diaz-Torres A., Thompson I.J. and Beck C., Phys. Rev. C, 68 (2003) 044607.

[20] Hashimoto S., Ogata K., Chiba S. and Yahiro M, Prog. Theor. Phys., 122 (2009) 1291. 